Supporting Information

\title{
Towards a total Synthesis of the novel polyketide natural product Spiculoic Acid A
}

\author{
Goverdhan Mehta,* Uday Kumar Kundu
}

Department of Organic Chemistry, Indian Institute of Science, Bangalore 560012, INDIA. gm@orgchem.iisc.ernet.in 
$(-)-16:$

$[\alpha]_{\mathrm{D}}^{24}=-10.0\left(c 1.2, \mathrm{CHCl}_{3}\right)$.

IR (neat) $3435,2964,1740,1243,1037 \mathrm{~cm}^{-1}$.

${ }^{1} \mathrm{H}$ NMR $\left(300 \mathrm{MHz}, \mathrm{CDCl}_{3}\right): \delta$ 4.14-4.01 (m, 2H), 3.57-3.45 (m,2H), $2.06(\mathrm{~s}, 3 \mathrm{H}), 2.01-$ $1.95(\mathrm{~m}, 1 \mathrm{H}), 0.95(\mathrm{~d}, J=6.9 \mathrm{~Hz}, 3 \mathrm{H})$.

${ }^{13} \mathrm{C}$ NMR (75MHz, $\left.\mathrm{CDCl}_{3}\right): 171.6,66.1,64.3,35.3,20.8,13.4$.

HRMS (ES) $m / z$ calcd for $\mathbf{C}_{6} \mathbf{H}_{\mathbf{1 2}} \mathbf{O}_{\mathbf{3}} \mathbf{N a}(\mathrm{M}+\mathrm{Na})^{+}: 155.0684$, found 155.0683 .

$(-)-18:$

$[\alpha]_{\mathrm{D}}{ }^{24}=-1.57\left(c 8.87, \mathrm{CHCl}_{3}\right)$.

IR (neat) 2952, 2885, 1725, 1658, $1043 \mathrm{~cm}^{-1}$.

${ }^{1} \mathrm{H}$ NMR $\left(300 \mathrm{MHz}, \mathrm{CDCl}_{3}\right): \delta 6.94(\mathrm{dd}, J=7.5 \mathrm{~Hz} 16.2 \mathrm{~Hz}, 1 \mathrm{H}), 5.88(\mathrm{~d}, J=16.8 \mathrm{~Hz}, 1 \mathrm{H})$ $4.58(\mathrm{~s}, 2 \mathrm{H}), 3.70(\mathrm{~s}, 3 \mathrm{H}), 3.45(\mathrm{~d}, J=6.3 \mathrm{~Hz}, 2 \mathrm{H}), 3.32(\mathrm{~s}, 3 \mathrm{H}), 2.63-2.51(\mathrm{~m}, 1 \mathrm{H}), 1.07(\mathrm{~d}$, $J=6.6 \mathrm{~Hz}, 3 \mathrm{H})$.

${ }^{13} \mathrm{C}$ NMR (75MHz, $\left.\mathrm{CDCl}_{3}\right): 166.9,155.2,120.6,96.4,71.3,55.2,51.3,36.6,15.9$.

HRMS (ES) $m / z$ calcd for $\mathbf{C}_{\mathbf{9}} \mathbf{H}_{16} \mathbf{O}_{\mathbf{4}} \mathbf{N a}(\mathrm{M}+\mathrm{Na})^{+}: 211.0946$, found 211.0952 .

$(-)-19:$

$[\alpha]_{\mathrm{D}}^{25}=-6.19\left(c 1.13, \mathrm{CHCl}_{3}\right)$.

IR (neat) $3418,2931,2875,1109,1042 \mathrm{~cm}^{-1}$.

${ }^{1} \mathrm{H}$ NMR $\left(300 \mathrm{MHz}, \mathrm{CDCl}_{3}\right): \delta$ 5.69-5.65 (m, 2H), $4.60(\mathrm{~s}, 2 \mathrm{H}), 3.42-3.38(\mathrm{~m}, 2 \mathrm{H}), 3.34$ $(\mathrm{s}, 3 \mathrm{H}), 2.51-2.42(\mathrm{~m}, 1 \mathrm{H}), 1.67(\mathrm{brs}, 1 \mathrm{H}), 1.03(\mathrm{~d}, J=6.9 \mathrm{~Hz}, 3 \mathrm{H})$.

${ }^{13} \mathrm{C}$ NMR $\left(75 \mathrm{MHz}, \mathrm{CDCl}_{3}\right)$ : 135.0, 128.9, 96.4, 72.4, 63.6, 55.1, 36.4, 16.8.

HRMS (ES) $m / z$ calcd for $\mathbf{C}_{\mathbf{8}} \mathbf{H}_{\mathbf{1 6}} \mathbf{O}_{\mathbf{3}} \mathbf{N a}(\mathrm{M}+\mathrm{Na})^{+}:$183.0997, found 183.0994.

$(-)-21:$

$[\alpha]_{\mathrm{D}}^{27}=-10.4\left(c 1.64, \mathrm{CHCl}_{3}\right)$.

IR (neat) $3045,2963,2930,1040 \mathrm{~cm}^{-1}$.

${ }^{1} \mathrm{H}$ NMR $\left(300 \mathrm{MHz}, \mathrm{CDCl}_{3}\right): \delta 4.63(\mathrm{~s}, 2 \mathrm{H}), 3.97-3.93(\mathrm{~m}, 2 \mathrm{H}), 3.78-3.54(\mathrm{~m}, 4 \mathrm{H}), 3.38$ $(\mathrm{s}, 3 \mathrm{H}), 3.33$ (brs, $1 \mathrm{H}), 2.13-2.09(\mathrm{~m}, 1 \mathrm{H}), 1.62-1.49(\mathrm{~m}, 3 \mathrm{H}), 1.00(\mathrm{t}, J=8.7 \mathrm{~Hz}, 3 \mathrm{H})$, $0.91,(\mathrm{~d}, J=6.9 \mathrm{~Hz}, 3 \mathrm{H})$.

${ }^{13} \mathrm{C}$ NMR (75MHz, $\mathrm{CDCl}_{3}$ ): 96.6, 80.5, 72.7, 62.9, 55.6, 42.8, 35.8, 21.6, 14.1, 11.8.

HRMS (ES) $m / z$ calcd for $\mathbf{C}_{\mathbf{1 0}} \mathbf{H}_{\mathbf{2} 2} \mathbf{O}_{\mathbf{4}} \mathbf{N a}(\mathrm{M}+\mathrm{Na})^{+}: 229.1416$, found 229.1424 .

(+)-23:

$[\alpha]_{\mathrm{D}}^{25}=14.2\left(c 0.70, \mathrm{CHCl}_{3}\right)$.

IR (neat) 3497, 2933, 1711, 1221, $1045 \mathrm{~cm}^{-1}$.

${ }^{1} \mathrm{H}$ NMR $\left(300 \mathrm{MHz}, \mathrm{CDCl}_{3}\right): \delta 6.77(\mathrm{~d}, J=10.8 \mathrm{~Hz}, 1 \mathrm{H}), 4.61(\mathrm{~s}, 2 \mathrm{H}), 4.19(\mathrm{dq}, J=$ $1.6 \mathrm{~Hz}, 7.5 \mathrm{~Hz}, 2 \mathrm{H}), 3.70-3.47(\mathrm{~m}, 3 \mathrm{H}), 3.37(\mathrm{~s}, 3 \mathrm{H}), 3.21(\mathrm{~d}, J=3 \mathrm{~Hz}, 1 \mathrm{H}), 2.50-2.29(\mathrm{~m}$, $3 \mathrm{H}), 1.84-1.38(\mathrm{~m}, 3 \mathrm{H}), 1.29(\mathrm{t}, J=6.9 \mathrm{~Hz}, 3 \mathrm{H}), 1.03(\mathrm{t}, J=7.2 \mathrm{~Hz}, 3 \mathrm{H}), 0.92-0.083(\mathrm{~m}$, $6 \mathrm{H})$.

${ }^{13} \mathrm{C}$ NMR $\left(75 \mathrm{MHz}, \mathrm{CDCl}_{3}\right): 167.8,141.1,135.2,96.7,77.4,72.5,60.3,55.5,43.5,36.4$, $24.9,20.6,14.3,14.1,13.9,12.1$.

HRMS (ES) $m / z$ calcd for $\mathbf{C}_{\mathbf{1 6}} \mathbf{H}_{\mathbf{3 0}} \mathbf{O}_{\mathbf{5}} \mathbf{N a}(\mathrm{M}+\mathrm{Na})^{+}: 325.1991$, found 325.2001 .

$(+)-27:$

$[\alpha]_{\mathrm{D}}^{25}=17.39\left(c 2.53, \mathrm{CHCl}_{3}\right)$.

IR (neat) $3408,2962,2887,1456,1041 \mathrm{~cm}^{-1}$. 
${ }^{1} \mathrm{H}$ NMR $\left(300 \mathrm{MHz}, \mathrm{CDCl}_{3}\right): \delta$ 7.70-7.67(m, 4H), 7.43-7.27 (m, 11H), $5.48(\mathrm{~d}, J=10.2$ $\mathrm{Hz}, 1 \mathrm{H}), 4.70\left[\mathrm{~d}_{(1 / 2 \mathrm{AB}) \mathrm{q}}, J=11.1 \mathrm{~Hz}, 1 \mathrm{H}\right], 4.57\left[\mathrm{~d}_{(1 / 2 \mathrm{AB}) \mathrm{q}} \mathrm{d}, J=11.1 \mathrm{~Hz}, 1 \mathrm{H}\right], 4.14(\mathrm{~s}, 2 \mathrm{H})$, 3.73-3.62 (m, 2H), 3.38-3.34 (m, 1H), $2.73($ brs, $1 \mathrm{H}), 2.61-2.52(\mathrm{~m}, 1 \mathrm{H}), 2.16-2.02(\mathrm{~m}$, $2 \mathrm{H}), 1.95-1.87(\mathrm{~m}, 1 \mathrm{H}), 1.60-1.30(\mathrm{~m}, 2 \mathrm{H}), 1.09-1.05(\mathrm{~m}, 9 \mathrm{H}), 0.98-0.88(\mathrm{~m}, 9 \mathrm{H})$.

${ }^{13} \mathrm{C}$ NMR (75MHz, $\left.\mathrm{CDCl}_{3}\right): 141.3,138.3,135.6,135.5,133.9,129.5,128.3,127.6,127.5$, 124.5, 88.2, 75.1, 66.7, 66.3, 42.6, 37.6, 26.8, 25.4, 21.4, 19.2, 15.4, 13.1, 12.3 .

HRMS (ES) $m / z$ calcd for $\mathbf{C}_{35} \mathbf{H}_{48} \mathbf{O}_{3} \mathbf{S i N a}(\mathrm{M}+\mathrm{Na})^{+}: 567.3270$, found 567.3274 .

(+)-31:

$[\alpha]_{\mathrm{D}}^{25}=26.2\left(\mathrm{c} 2.06, \mathrm{CHCl}_{3}\right)$.

IR (neat) 2961, 2930, 1725, $1110 \mathrm{~cm}^{-1}$.

${ }^{1} \mathrm{H}$ NMR $\left(300 \mathrm{MHz}, \mathrm{CDCl}_{3}\right): \delta$ 7.69-7.66 (m, 5H), 7.42-7.26 (m, 10H), $7.12(\mathrm{dd}, J=8.4$ $\mathrm{Hz}, 15.6 \mathrm{~Hz}, 1 \mathrm{H}), 5.80(\mathrm{~d}, J=15.6 \mathrm{~Hz}, 1 \mathrm{H}), 5.45(\mathrm{~d}, J=10.2 \mathrm{~Hz}, 1 \mathrm{H}), 4.60\left(\mathrm{~d}_{1 / 2 \mathrm{ABq}}, J=\right.$ $11.4 \mathrm{~Hz}, 1 \mathrm{H}), 4.50\left(\mathrm{~d}_{1 / 2 \mathrm{ABq}}, J=11.4 \mathrm{~Hz}, 1 \mathrm{H}\right), 4.13(\mathrm{~s}, 2 \mathrm{H}), 3.72(\mathrm{~s}, 3 \mathrm{H}), 3.26-3.22(\mathrm{~m}$, $1 \mathrm{H}), 2.64-2.44(\mathrm{~m}, 2 \mathrm{H}), 2.13-1.99(\mathrm{~m}, 2 \mathrm{H}), 1.07-1.04(\mathrm{~m}, 12 \mathrm{H}), 0.93-0.82(\mathrm{~m}, 8 \mathrm{H})$.

${ }^{13} \mathrm{C}$ NMR (75MHz, $\left.\mathrm{CDCl}_{3}\right): 167.2,152.3,141.2,138.7,135.5,133.9,129.5,128.1,127.6$, $127.5,127.3,124.6,120.4,85.9,74.3,66.8,51.3,42.4,40.0,26.8,25.2,21.3,19.3,17.0$, 13.0, 12.2 .

LRMS (ES) $m / z$ calcd for $\mathbf{C}_{\mathbf{3 8}} \mathbf{H}_{50} \mathbf{O}_{4} \mathrm{SiNa}(\mathrm{M}+\mathrm{Na})^{+}:$: 621, found 621.

(+)-33:

$[\alpha]_{\mathrm{D}}{ }^{27}=20.133\left(c 4.52, \mathrm{CHCl}_{3}\right)$.

IR (neat) $2961,2857,1725,1428,1270 \mathrm{~cm}^{-1}$.

${ }^{1} \mathrm{H}$ NMR $\left(300 \mathrm{MHz}, \mathrm{CDCl}_{3}\right): \delta 9.45(\mathrm{~d}, J=8.1 \mathrm{~Hz}, 1 \mathrm{H}), 7.68-7.66(\mathrm{~m}, 4 \mathrm{H}), 7.43-7.25(\mathrm{~m}$, $11 \mathrm{H}), 7.01(\mathrm{dd}, J=7.8 \mathrm{~Hz}, 15.6 \mathrm{~Hz}, 1 \mathrm{H}), 6.09(\mathrm{dd}, J=7.5 \mathrm{~Hz}, 15.6 \mathrm{~Hz}, 1 \mathrm{H}), 5.46(\mathrm{~d}, J=$ $10.2 \mathrm{~Hz}, 1 \mathrm{H}), 4.64\left[\mathrm{~d}_{(1 / 2 \mathrm{AB}) \mathrm{q}}, J=10.8 \mathrm{~Hz}, 1 \mathrm{H}\right], 4.48\left[\mathrm{~d}_{(1 / 2 \mathrm{AB}) \mathrm{q}}, J=11.1 \mathrm{~Hz}, 1 \mathrm{H}\right], 4.13(\mathrm{~s}$, $2 \mathrm{H}), 3.30(\mathrm{t}, J=4.8 \mathrm{~Hz}, 1 \mathrm{H}), 2.76-2.74(\mathrm{~m}, 1 \mathrm{H}), 2.51-2.45(\mathrm{~m}, 1 \mathrm{H}), 2.11-1.94(\mathrm{~m}, 2 \mathrm{H})$, $1.36-1.28(\mathrm{~m}, 2 \mathrm{H}), 1.15-1.05(\mathrm{~m}, 12 \mathrm{H})$ 0.92-0.83 (m, 6H).

${ }^{13} \mathrm{C}$ NMR $\left(75 \mathrm{MHz}, \mathrm{CDCl}_{3}\right): 194.3,161.8,141.5,138.5,135.5,133.8,132.2,129.5,128.2$, 127.6, 127.5, 124.2, 86.0, 74.5, 66.6, 42.8, 40.3, 26.8, 25.0, 21.4, 19.2, 17.3, 13.0, 12.1 .

HRMS (ES) $m / z$ calcd for $\mathbf{C}_{\mathbf{3 7}} \mathbf{H}_{\mathbf{4 8}} \mathbf{O}_{\mathbf{3}} \mathbf{S i N a}(\mathrm{M}+\mathrm{Na})^{+}:$591.3270, found 591.3268.

(+)-34:

$[\alpha]_{\mathrm{D}}^{26}=33.8\left(c 0.62, \mathrm{CHCl}_{3}\right)$.

IR (neat) 2956, 2926, 1721, 1589, 1436, $1118 \mathrm{~cm}^{-1}$.

${ }^{1} \mathrm{H}$ NMR $\left(400 \mathrm{MHz}, \mathrm{CDCl}_{3}\right): \delta 7.67(\mathrm{~d}, J=7.8 \mathrm{~Hz}, 4 \mathrm{H}), 7.38-7.23(\mathrm{~m}, 12 \mathrm{H}), 6.27-6.10$ $(\mathrm{m}, 2 \mathrm{H}), \quad 5.76(\mathrm{~d}, J=15.6 \mathrm{~Hz}, 1 \mathrm{H}), 5.45(\mathrm{~d}, J=10.4 \mathrm{~Hz}, 1 \mathrm{H}), 4.57\left[\mathrm{~d}_{(1 / 2 \mathrm{AB}) \mathrm{q}}, J=11.2\right.$ $\mathrm{Hz}, 1 \mathrm{H}), 4.48\left[\mathrm{~d}_{(1 / 2 \mathrm{AB}) \mathrm{q}}, J=11.2 \mathrm{~Hz}, 1 \mathrm{H}\right), 4.13(\mathrm{~s}, 2 \mathrm{H}), 3.73(\mathrm{~s}, 3 \mathrm{H}), 3.22-3.19(\mathrm{~m}, 1 \mathrm{H})$, 2.58-2.44 (m, 2H), 2.11-2.00 (m, 2H), 1.32-1.21 (m, 2H), 1.06-1.04 (m, 12H), $0.91(\mathrm{t}, J=$ $7.6 \mathrm{~Hz}, 3 \mathrm{H}), 0.84(\mathrm{t}, J=7.2 \mathrm{~Hz}, 3 \mathrm{H})$.

${ }^{13} \mathrm{C}$ NMR $\left(100 \mathrm{MHz}, \mathrm{CDCl}_{3}\right): 167.7,147.5,145.5,141.0,138.9,135.6,135.5,134.0$, $133.9,129.5,128.2,127.9,127.6,127.5,127.3,124.9,118.9,86.2,74.3,66.9,51.4,42.5$, $40.5,26.8,25.1,21.4,19.3,17.6,13.0,12.2$.

HRMS (ES) $m / z$ calcd for $\mathbf{C}_{40} \mathbf{H}_{52} \mathbf{O}_{4} \mathbf{S i N a}(\mathrm{M}+\mathrm{Na})^{+}:$647.3533, found 647.3543 . 
$(-)-9:$

$[\alpha]_{\mathrm{D}}^{26}=-50\left(c 0.60, \mathrm{CHCl}_{3}\right)$.

IR (neat) 2929, 2856, 1737, $1589 \mathrm{~cm}^{-1}$

${ }^{1} \mathrm{H}$ NMR $\left(400 \mathrm{MHz}, \mathrm{CDCl}_{3}\right): \delta$ 7.65-7.59 $(\mathrm{m}, 4 \mathrm{H}), 7.42-7.24(\mathrm{~m}, 11 \mathrm{H}), 5.60(\mathrm{dd}, J=2$ $\mathrm{Hz}, 10 \mathrm{~Hz}, 1 \mathrm{H}), 5.41(\mathrm{ddd}, J=1.6 \mathrm{~Hz}, 4.8 \mathrm{~Hz}, 10 \mathrm{~Hz}, 1 \mathrm{H}), 4.59\left[\mathrm{~d}_{(1 / 2 \mathrm{AB}) \mathrm{q}}, J=11.6 \mathrm{~Hz}, 1 \mathrm{H}\right]$, $4.47\left[\mathrm{~d}_{(1 / 2 \mathrm{AB}) \mathrm{q}}, J=11.6 \mathrm{~Hz}, 1 \mathrm{H}\right], 3.87-3.84(\mathrm{~m}, 1 \mathrm{H}), 3.60(\mathrm{~s}, 3 \mathrm{H}), 3.44\left[\mathrm{~d}_{(1 / 2 \mathrm{AB}) \mathrm{q}}, J=10 \mathrm{~Hz}\right.$, $1 \mathrm{H}], 3.39\left[\mathrm{~d}_{(1 / 2 \mathrm{AB}) \mathrm{q}}, J=10 \mathrm{~Hz}, 1 \mathrm{H}\right], 3.18(\mathrm{~d}, J=4.8 \mathrm{~Hz}, 1 \mathrm{H}), 2.26-2.25(\mathrm{~m}, 1 \mathrm{H}), 2.13(\mathrm{t}, J$ $=8.8 \mathrm{~Hz}, 1 \mathrm{H}), 2.00-1.90(\mathrm{~m}, 2 \mathrm{H}), 1.67-1.62(\mathrm{~m}, 2 \mathrm{H}), 1.46-1.40(\mathrm{~m}, 2 \mathrm{H}), 1.07-1.00(\mathrm{~m}$, $12 \mathrm{H}), 0.90-0.81(\mathrm{~m}, 6 \mathrm{H})$.

${ }^{13} \mathrm{C}$ NMR $\left(100 \mathrm{MHz}, \mathrm{CDCl}_{3}\right): 174.0,139.7,135.8,133.8,133.7,133.1,129.5,128.1$, 127.6, 127.5, 127.2, 127.1, 121.7, 83.4, 73.2, 66.8, 54.3, 45.6, 45.5, 43.9, 43.5, 43.1, 42.7, 27.0, 25.2, 24.4, 19.4, 14.3, 14.1, 8.1.

HRMS (ES) $m / z$ calcd for $\mathbf{C}_{\mathbf{4 0}} \mathbf{H}_{52} \mathbf{O}_{4} \mathbf{S i N a}(\mathrm{M}+\mathrm{Na})^{+}:$647.3533, found 647.3564 . 

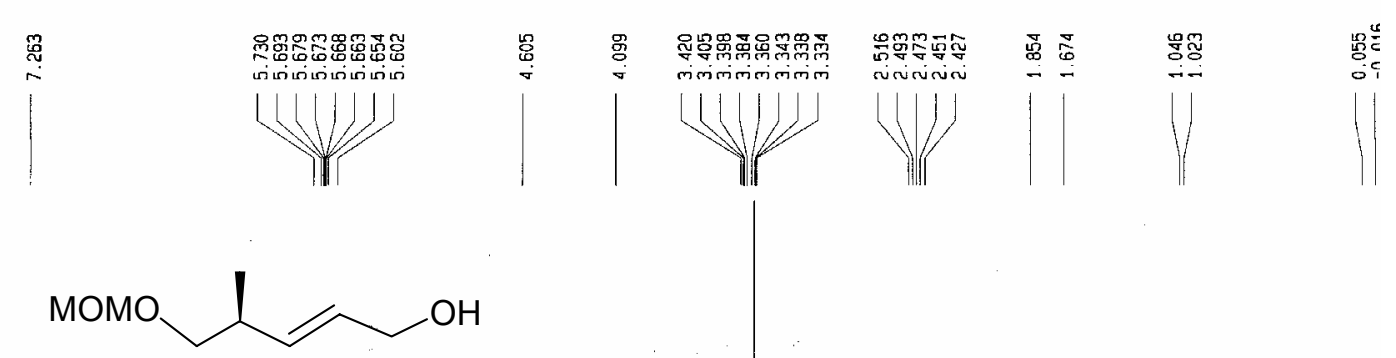

(-) 19
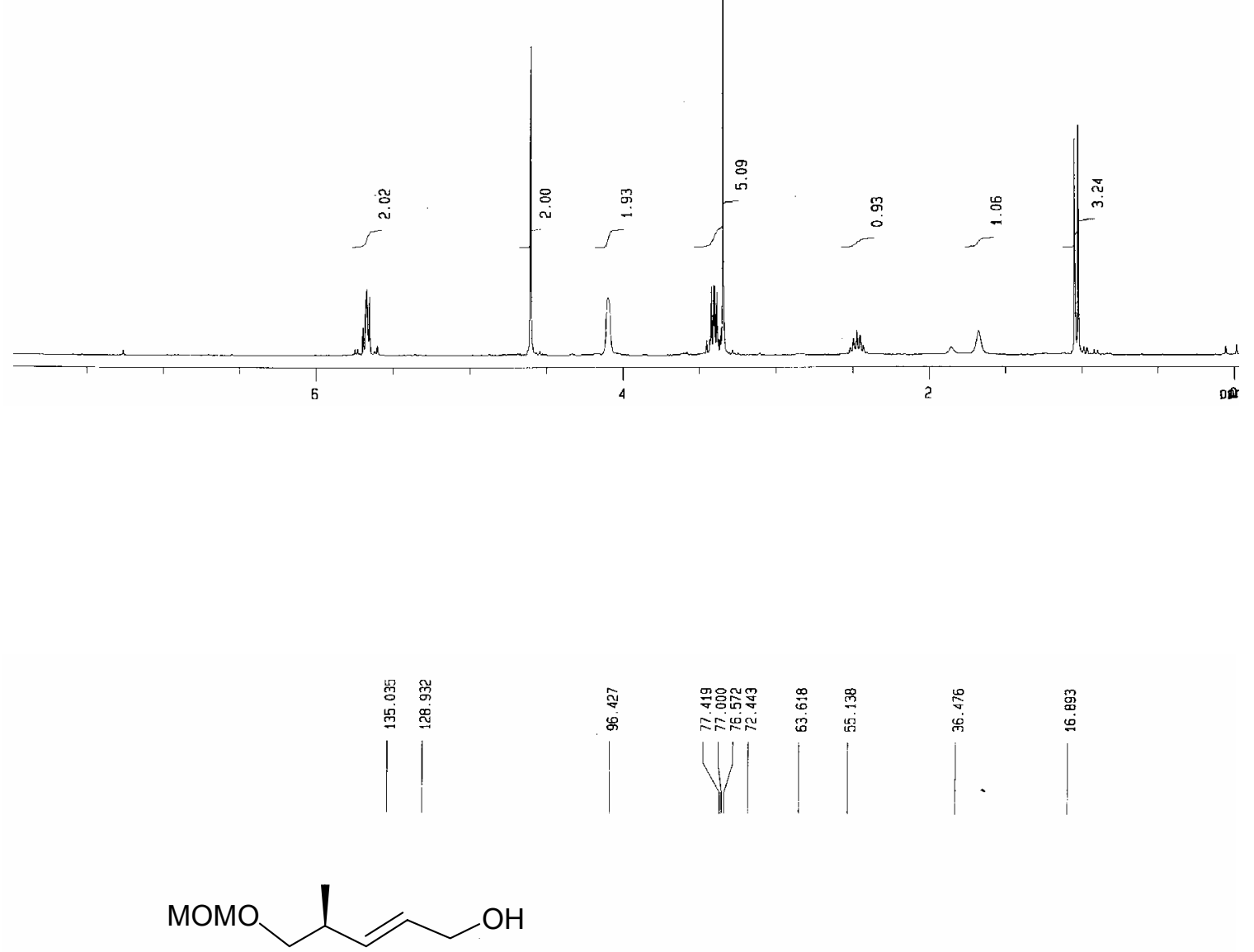

(-) 19

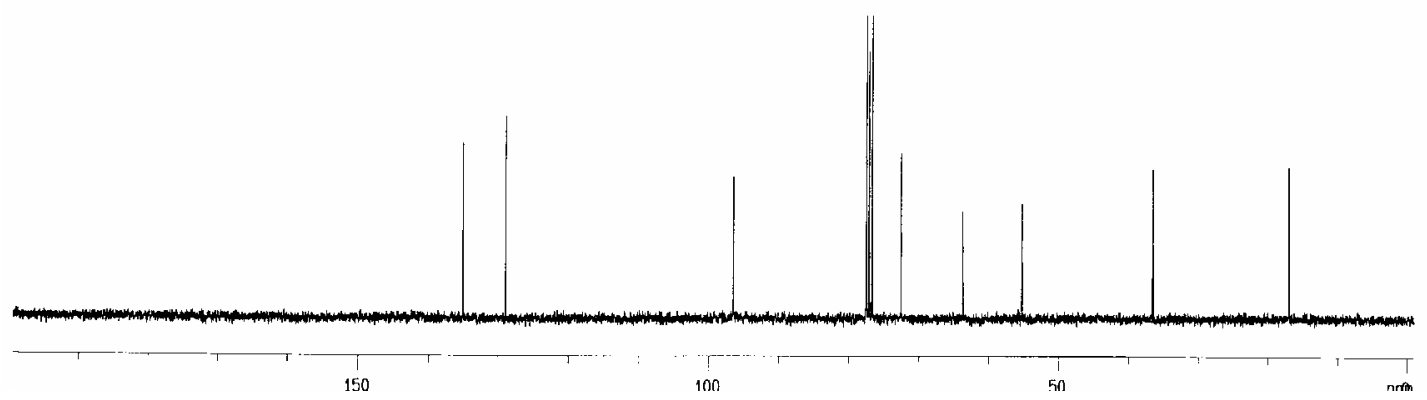



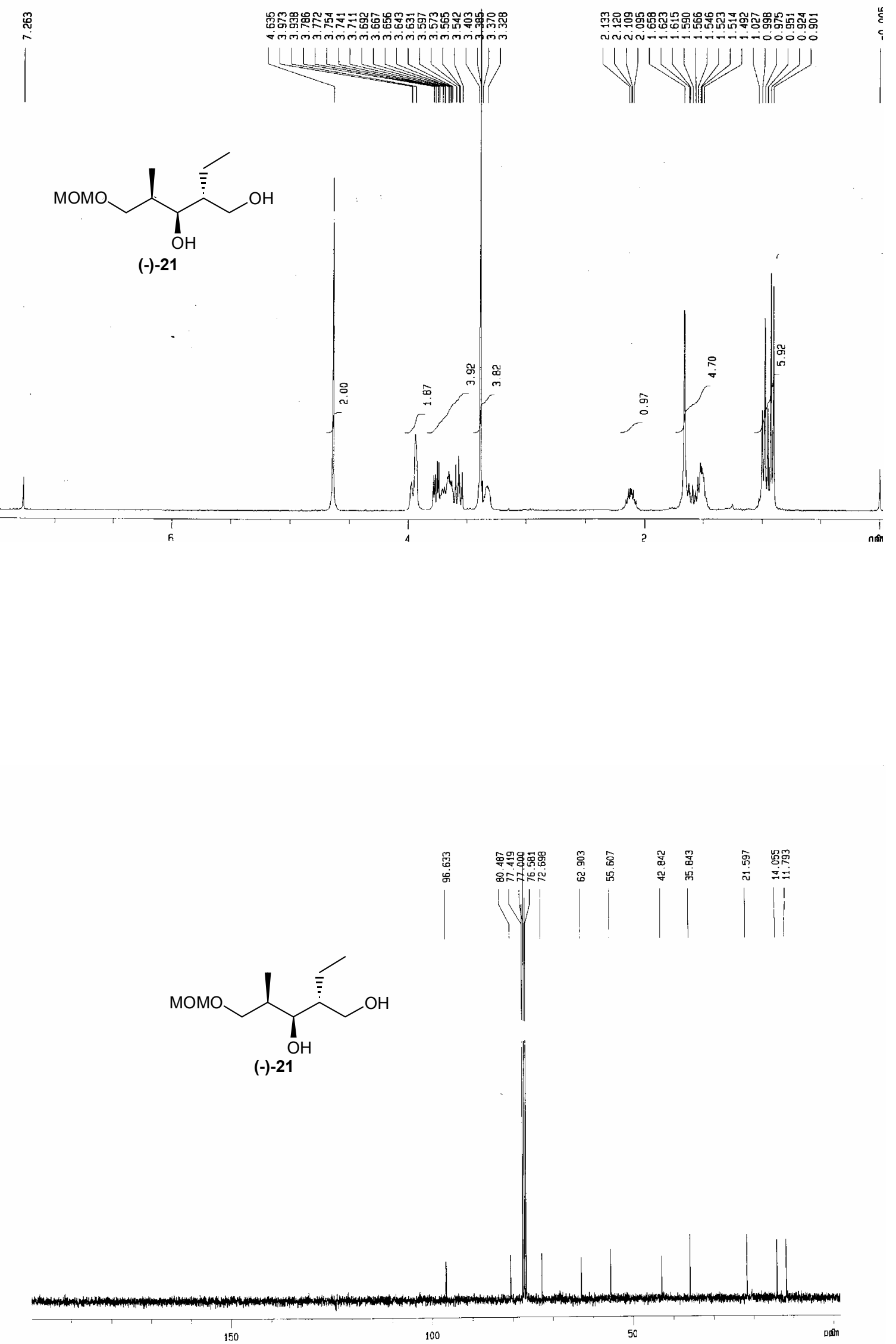

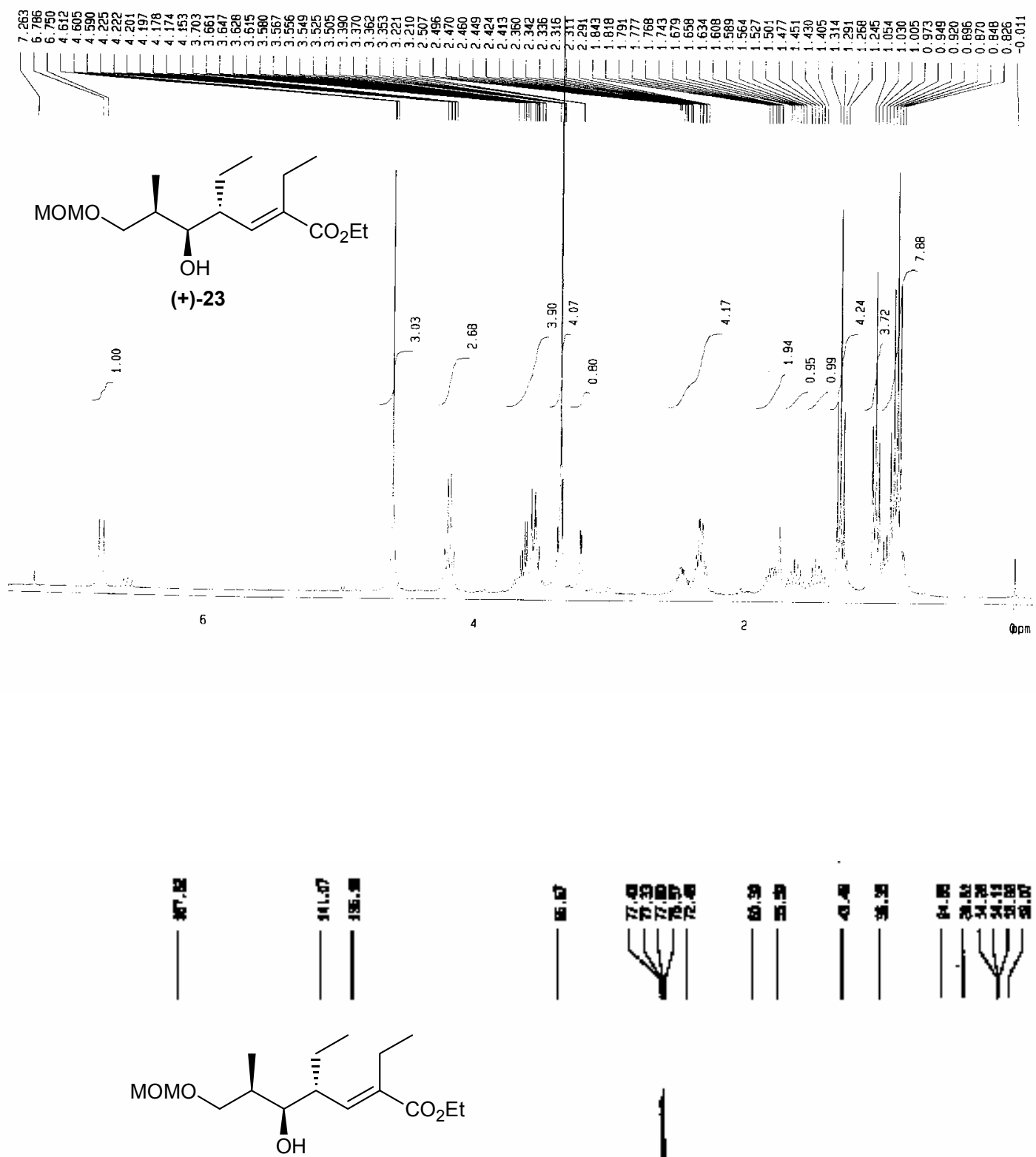

(+)-23

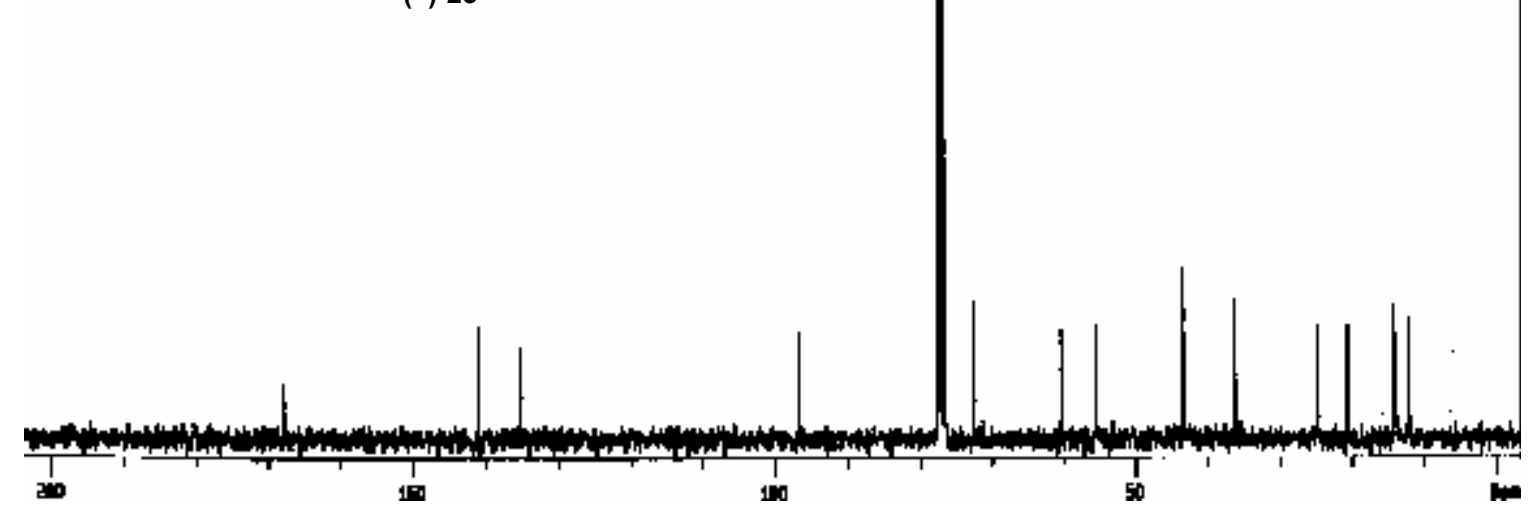



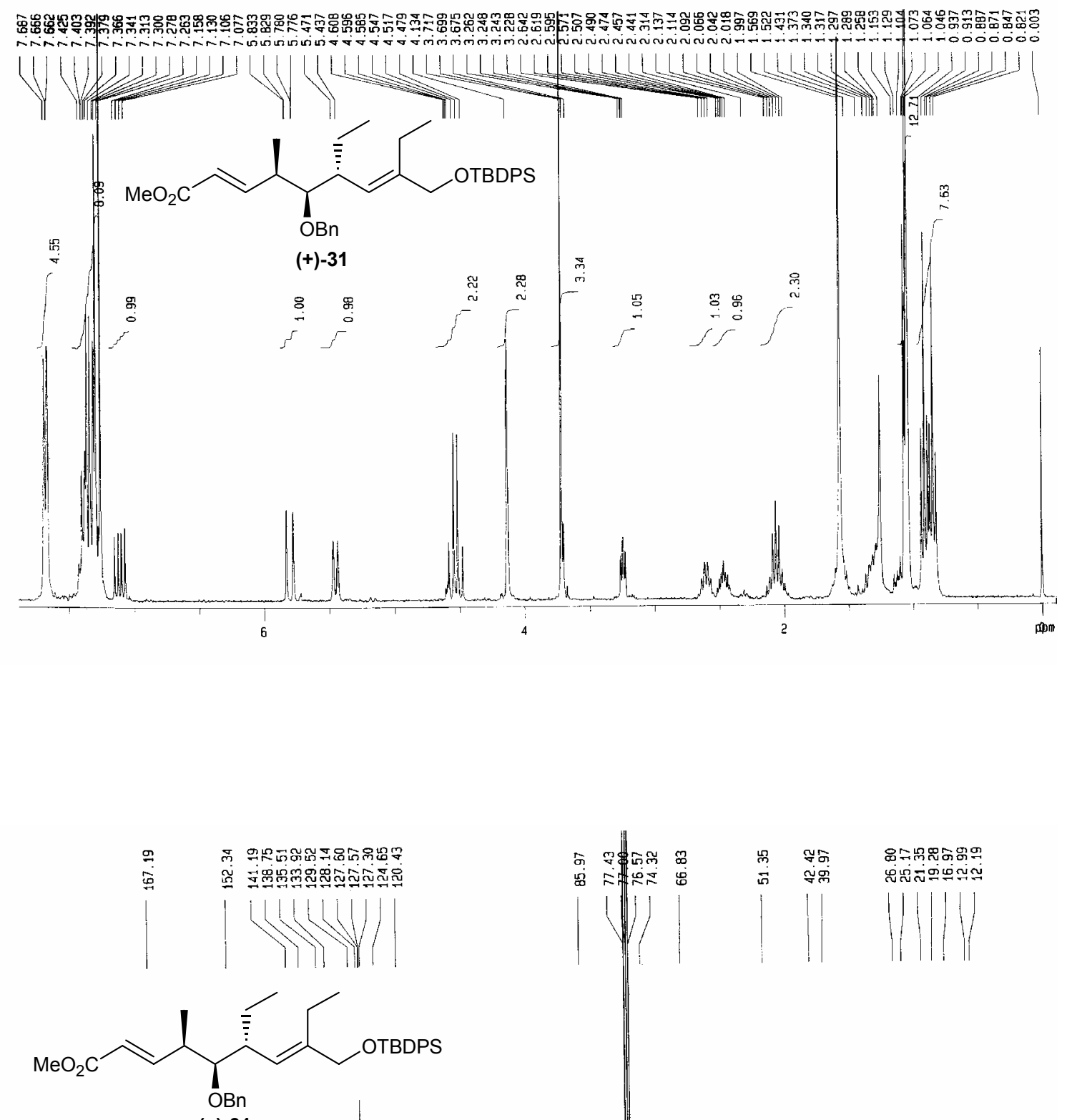

(+)-31

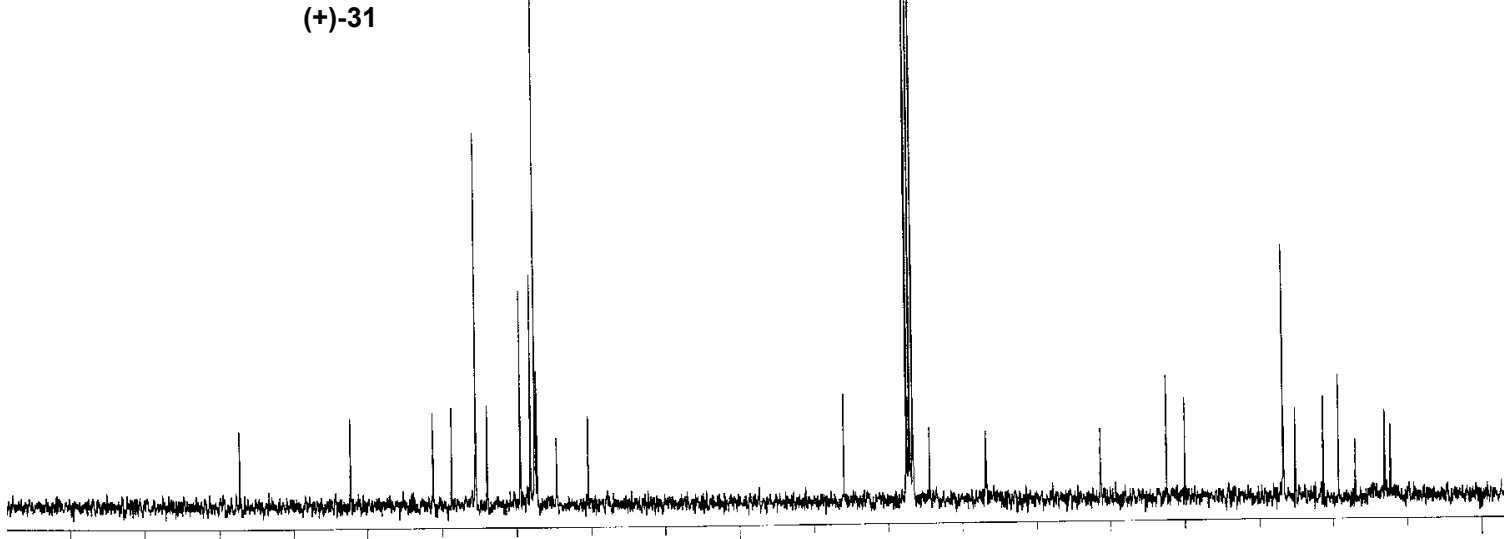



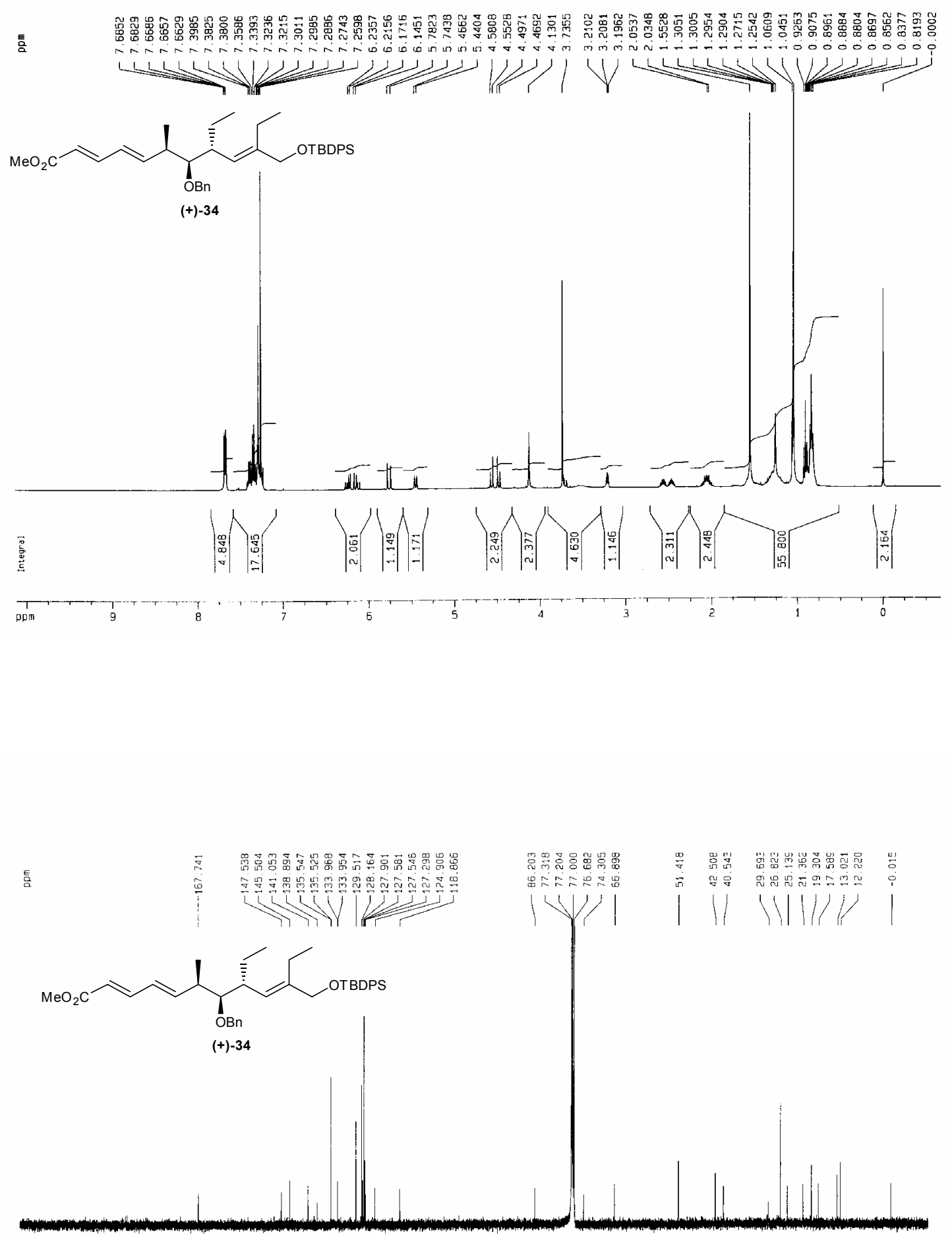

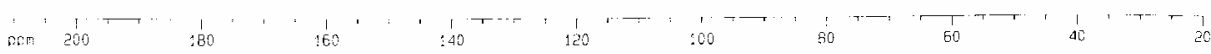



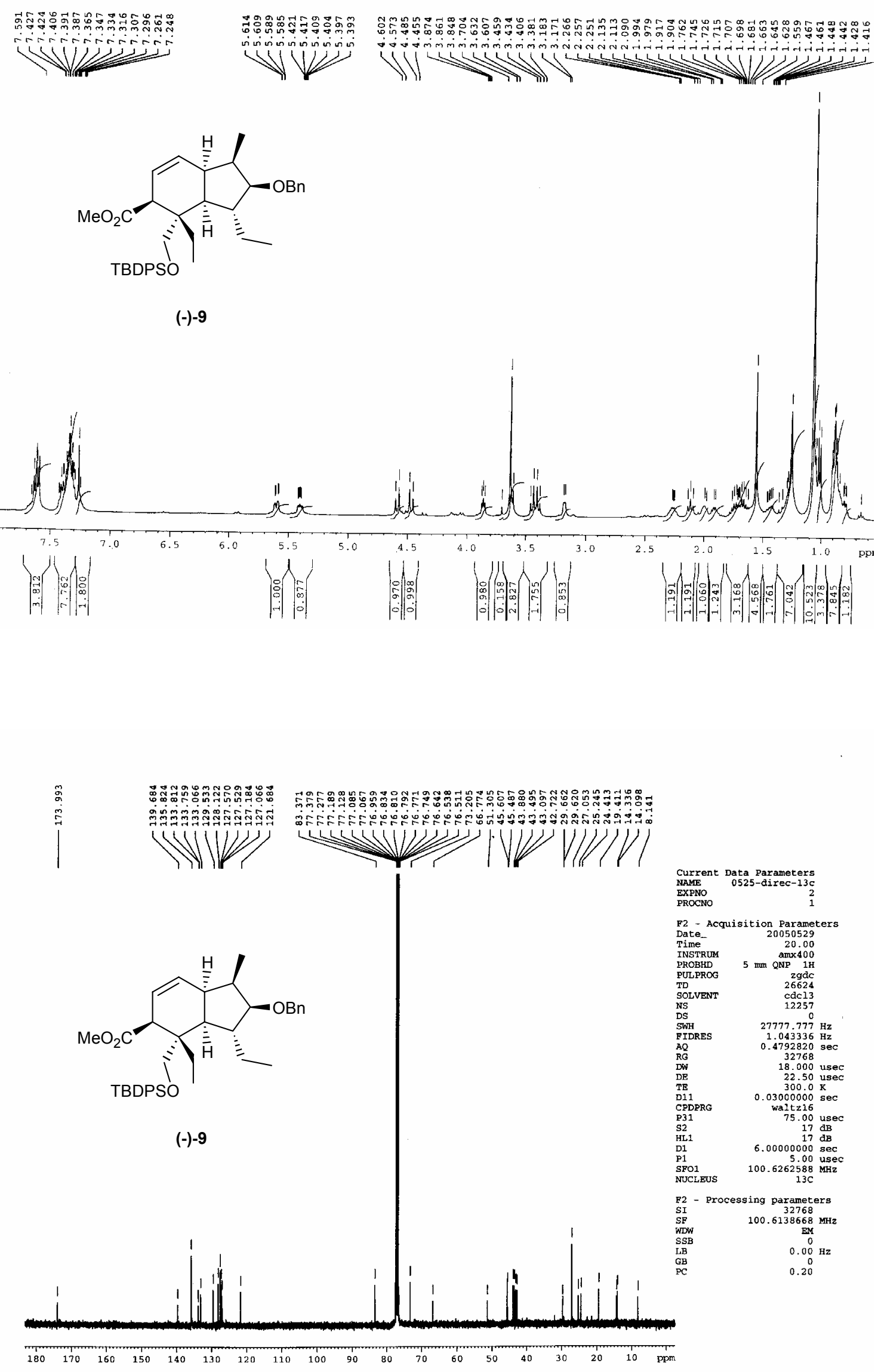


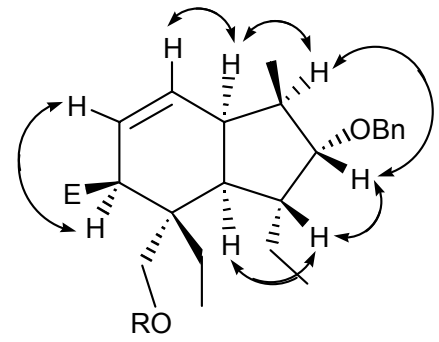

${ }^{1} \mathrm{H}-{ }^{1} \mathrm{H}$ COSY corelations in (-)-9
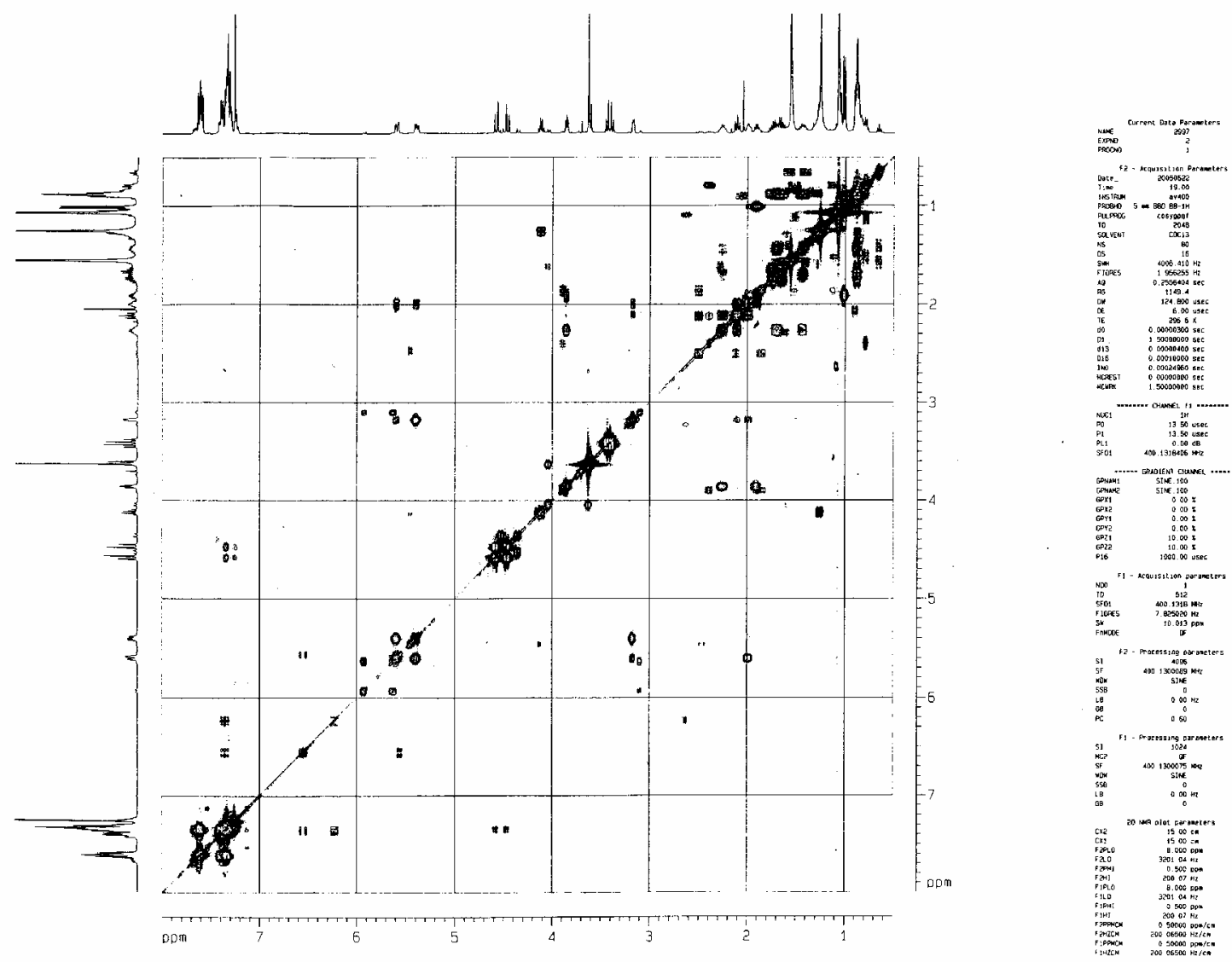


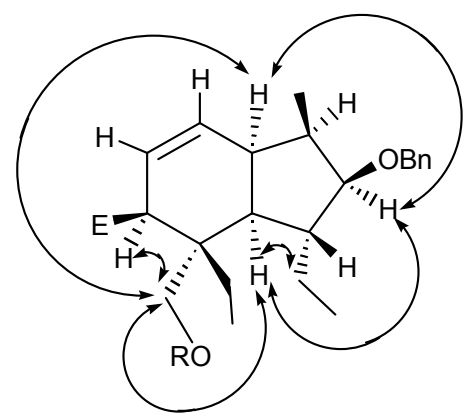

${ }^{1} \mathrm{H}-{ }^{1} \mathrm{H}$ nOe corelations in (-)-9
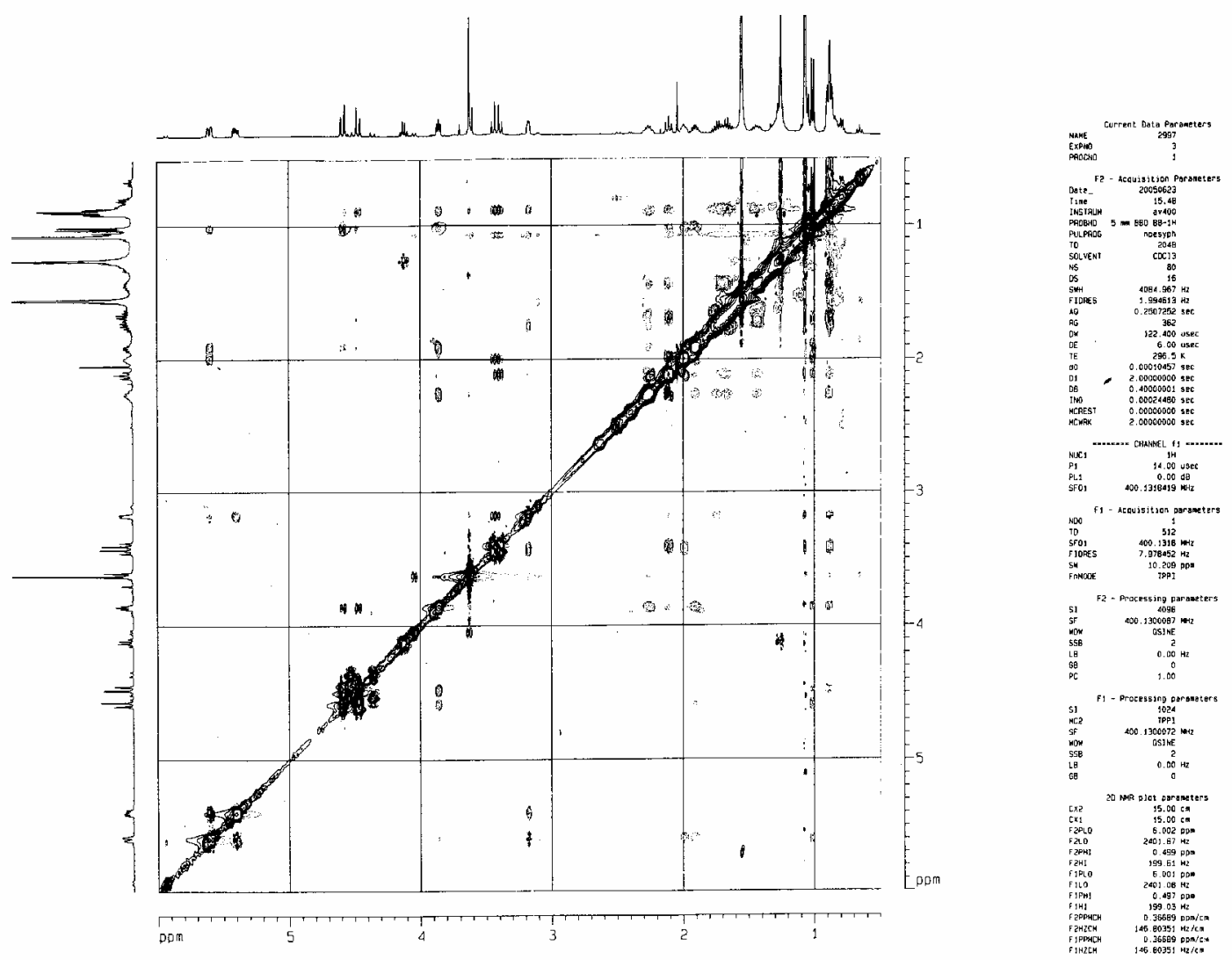